\title{
Correction
}

\section{Correction to: Pseudorandom number generator based on a 5D hyperchaotic four-wing memristive system and its FPGA implementation}

Fei $\mathrm{Yu}^{1,2, a}$, Lixiang $\mathrm{Li}^{1}$, Binyong $\mathrm{He}^{1}$, Li Liu ${ }^{1}$, Shuai Qian ${ }^{1}$, Zinan Zhang ${ }^{1}$, Hui Shen ${ }^{1}$, Shuo $\mathrm{Cai}^{1}$, and $\mathrm{Yi} \mathrm{Li}^{3}$

${ }^{1}$ School of Computer and Communication Engineering, Changsha University of Science and Technology, Changsha 410114, China

${ }^{2}$ Guangxi Key Laboratory of Cryptography and Information Security, Guilin University of Electronic Technology, Guilin 541004, China

${ }^{3}$ Hunan Post and Telecommunication Planning and Designing Institute, No. 236 Yuanda Road, Changsha 410126, China

Published online 24 June 2021

() EDP Sciences, Springer-Verlag GmbH Germany, part of Springer Nature 2021

Correction to: Eur. Phys. J. Spec. Top.

https://doi.org/10.1140/epjs/s11734-021-00132-x

Due to a typesetting mistake, the article was published with errors in four references.

The references have been corrected as follows:

[54]: S. Zhong, Computer. Mater. Continua 60, 465479 (2019)

[55]: H. Lin, C. Wang, Y. Sun et al., Nonlinear Dyn. 100, 3667-3683 ( 2020)
[59]: F. Yu, L. Li, B. He et al., IEEE Access 7, 181884-181898 (2019)

[60]: F. Yu, L. Liu, B. He et al., Complexity 2019, 4047957 (2019)

No other changes to the article have been made. We apologise for any inconvenience caused to our readers.

The original article can be found online at https://doi.org/ 10.1140/epjs/s11734-021-00132-x.

\footnotetext{
${ }^{\mathrm{a}}$ e-mail: yufeiyfyf@csust.edu.cn (corresponding author)
} 